\title{
A new peg-filgrastim biosimilar, mecapegfilgrastim for primary prophylaxis of chemotherapy-related neutropenia is now available
}

\author{
Umberto Tirelli $^{1}$, Antonino Carbone ${ }^{1}$, Raffaele Di Francia ${ }^{2}$, Massimiliano Berretta $^{3}$ \\ ${ }^{1}$ Centro di Riferimento Oncologico, Istituto Nazionale Tumori, IRCCS, Aviano (PN), Italy; ${ }^{2}$ Italian Association of Pharmacogenomics and Molecular \\ Diagnostics (IAPharmagen), Ancona, Italy; ${ }^{3}$ Department of Medical Oncology, Centro di Riferimento Oncologico, Istituto Nazionale Tumori, \\ IRCCS, Aviano (PN), Italy \\ Correspondence to: Umberto Tirelli. Clinica Tirelli Medical Group Via Vallona 6833170 Pordenone, Italy. Email: utirelli@cro.it. \\ Provenance and Peer Review: This article was commissioned by the Editorial Office, Annals of Translational Medicine. The article did not undergo \\ external peer review. \\ Comment on: Xu F, Zhang Y, Miao Z, et al. Efficacy and safety of mecapegfilgrastim for prophylaxis of chemotherapy-induced neutropenia in patients \\ with breast cancer: a randomized, multicenter, active-controlled phase III trial. Ann Transl Med 2019;7:482.
}

Submitted Oct 05, 2019. Accepted for publication Oct 15, 2019.

doi: 10.21037/atm.2019.10.61

View this article at: http://dx.doi.org/10.21037/atm.2019.10.61

Chemotherapy is still one of the main tools for treatment of pediatric and adult blood and solid tumors and is capable of cure some acute leukemia, some advanced lymphomas, advanced testicular cancers among others (1). However, the main side effects and limiting factors for its use is myelotoxicity, in particular neutropenia and eventual life threatening febrile neutropenia. The recombinant human granulocyte colony stimulating factor, filgrastim, has been developed and employed in prevention of chemotherapyrelated neutropenia but because of its relatively short half-life, daily filgrastim injections were required to stimulate neutrophil recovery (2). Evaluation of several PEG-related forms of filgrastim identified PEG-filgrastim as the construct with optimal balance of in vitro activity and in vivo resistance time, is capable of self regulating clearance, remains in circulation during chemotherapyinduced neutropenia and is not eliminated until neutrophils start to recover (3-6). Clinical efficacy of PEG-filgrastim is achieved with a single $6 \mathrm{mg}$ dose once-per-chemotherapy cycle $(7,8)$. Therefore PEG-filgrastim is more convenient and less expensive for patients and hospitals, but in China PEG-filgrastim is not available leaving filgrastim to be the only treatment option in this setting, but with the need of daily injections. Mecapegfilgrastim is a biosimilar of PEGfilgrastim and has been developed by Jiangsu Hengrui Medicine Co,. Ltd. $(9,10)$ and has shown, in phase II studies in non small cell lung cancer (NSCLC) and in subsequent phase III study in the same tumors, to be effective as daily filgrastim prophylaxis of neutropenia-associated with chemotherapy and with comparable pharmacokinetics and safety profiles with PEG-filgrastim $(9,10)$. Considering the deficiency in long-acting anti-neutropenia drugs in China, and the limited data of mecapegfilgrastim in breast cancer patients, being only one phase II trial of mecapegfilgrastim performed in breast cancer (11), a randomized phase III trial was needed. In this very welldone study in breast cancer, $\mathrm{Xu}$ and co-workers (12) showed that in a randomized comparison of two different doses of Mecapegfilgrastim or filgrastim given during the first cycle of chemotherapy, Mecapegfilgrastim, when administered in primary prophylaxis of chemotherapy-induced neutropenia, to be non inferior and even superior to filgrastim. The fixed $6 \mathrm{mg}$ dose of Mecapegfilgrastim regimen showed similar efficacy and safety profile compared with the $100 \mu \mathrm{g} / \mathrm{kg}$ regimen of Meca and is preferable in clinical practice due to the convenient once per cycle administration and the high degree treatment compliance for the patient. This randomized study therefore provide new evidence for the novel long acting mecapegfilgrastim, to be a new safe and effective alternative for the prophylaxis of chemotherapyinduced neutropenia in comparison with filgrastim and can be used in a convenient one-per-cycle administration for breast cancer patients and possibly for other adult tumors. This new long acting PEG-filgrastim may be very useful also in some frail subgroups of patients like elderly patients $(13,14)$ and HIV-related tumors $(15-17)$, that fortunately 
are less common in China than in Western countries, in that they are more sensible and prone to bone marrow toxicity after chemotherapy. In the $\mathrm{Xu}$ et al. randomized study, the mean age of the 3 groups of patients was around $48 \pm 8$ years, therefore a quite young population, reflecting the epidemiology of breast cancer in China, in comparison with Western countries epidemiology. However, with the growing age in population occurring also in China, this long lasting PEG-filgrastim could be even more useful in elderly setting. The decreased cost due to the short period of hospitalization and the decreased risk of infection of mecapegfilgrastim with related limited costs, are both factors very important in the limited medical resources available now in all countries. It is also possible, but it is not yet known to us, also because mecapegfilgrastim it is not yet on the market, that this new mecapegfilgrastim by itself may cost less than filgrastim and other pegylated-filgrastim. Finally, for China but also for other countries, these results are very important in that allows the safe use with demonstrated activity of a new biosimilar of PEG-filgrastim, mecapegfilgrastim, developed in China. In addition, breast cancer is an ideal setting where this approach could be tested in that breast cancer is a very common malignancy and chemotherapy is still a very frequent and efficacious modality of treatment.

\section{Acknowledgments}

Funding: None.

\section{Footnote}

Conflicts of Interest: The authors have no conflicts of interest to declare.

Ethical Statement: The authors are accountable for all aspects of the work in ensuring that questions related to the accuracy or integrity of any part of the work are appropriately investigated and resolved.

Open Access Statement: This is an Open Access article distributed in accordance with the Creative Commons Attribution-NonCommercial-NoDerivs 4.0 International License (CC BY-NC-ND 4.0), which permits the noncommercial replication and distribution of the article with the strict proviso that no changes or edits are made and the original work is properly cited (including links to both the formal publication through the relevant DOI and the license).
See: https://creativecommons.org/licenses/by-nc-nd/4.0/.

\section{References}

1. Bonadonna G. Does chemotherapy fulfill its expectations in cancer treatment? Ann Oncol 1990;1:11-21.

2. Kuwabara T, Kobayashi S, Sugiyama Y. Pharmacokinetics and pharmacodynamics of a recombinant human granulocyte colony-stimulating factor. Drug Metab Rev 1996;28:625-58.

3. Balducci L, Al-Halawani H, Charu V, et al. Elderly cancer patients receiving chemotherapy benefit from first-cycle pegfilgrastim. Oncologist 2007;12:1416-24.

4. Hecht JR, Pillai M, Gollard R, et al. A randomized, placebo-controlled phase ii study evaluating the reduction of neutropenia and febrile neutropenia in patients with colorectal cancer receiving pegfilgrastim with every-2-week chemotherapy. Clin Colorectal Cancer 2010;9:95-101.

5. Burris HA, Belani CP, Kaufman PA, et al. Pegfilgrastim on the same day versus next day of chemotherapy in patients with breast cancer, non-small-cell lung cancer, ovarian cancer, and non-hodgkin's lymphoma: results of four multicenter, double-blind, randomized phase II studies. J Oncol Pract 2010;6:133-40.

6. Vogel CL, Wojtukiewicz MZ, Carroll RR, et al. First and subsequent cycle use of pegfilgrastim prevents febrile neutropenia in patients with breast cancer: a multicenter, double-blind, placebo-controlled phase III study. J Clin Oncol 2005;23:1178-84.

7. Yang BB, Kido A. Pharmacokinetics and pharmacodynamics of pegfilgrastim. Clin Pharmacokinet 2011;50:295-306.

8. Yang BB, Savin MA, Green M. Prevention of chemotherapy-induced neutropenia with pegfilgrastim: pharmacokinetics and patient outcomes. Chemotherapy 2012;58:387-98.

9. Yan B, Zhang W, Lu F, et al. Safety of polyethylene glycol recombinant human granulocyte colony-stimulating factor in treating non-small cell lung cancer patients at I $\mathrm{b}$ stage. Asian Pac J Trop Med 2013;6:912-5.

10. Zhou C, Huang Y, Wang D, et al. A Randomized Multicenter Phase III Study of Single Administration of Mecapegfilgrastim (HHPG-19K), a Pegfilgrastim Biosimilar, for Prophylaxis of Chemotherapy-Induced Neutropenia in Patients With Advanced Non-SmallCell Lung Cancer (NSCLC). Clin Lung Cancer 2016;17:119-27. 
11. Wang T, Wu B, Hu X, et al. A randomized multicenter phase II trial of mecapegfilgrastim single administration versus granulocyte colony-stimulating growth factor on treating chemotherapy-induced neutropenia in breast cancer patients. Ann Transl Med 2019;7:196.

12. Xu F, Zhang Y, Miao Z, et al. Efficacy and safety of mecapegfilgrastim for prophylaxis of chemotherapyinduced neutropenia in patients with breast cancer: a randomized, multicenter, active-controlled phase III trial. Ann Transl Med 2019;7:482.

13. Errante D, Vaccher E, Tirelli U. Are hematopoietic colony-stimulating factors useful in association with chemotherapy in the treatment of HIV-related nonHodgkin's lymphomas? Ann Oncol 1996;7:233-7.

14. Nasti G, Talamini R, Antinori A, et al. AIDS Clinical Trial Group Staging System in the Haart Era--the Italian Cooperative Group on AIDS and Tumors and the Italian Cohort of Patients Naive from Antiretrovirals. AIDS-

Cite this article as: Tirelli U, Carbone A, Di Francia R, Berretta M. A new peg-filgrastim biosimilar, mecapegfilgrastim for primary prophylaxis of chemotherapy-related neutropenia is now available. Ann Transl Med 2020;8(5):166. doi: 10.21037/ atm.2019.10.61 related Kaposi's Sarcoma: evaluation of potential new prognostic factors and assessment of the AIDS Clinical Trial Group Staging System in the Haart Era--the Italian Cooperative Group on AIDS and Tumors and the Italian Cohort of Patients Naive From Antiretrovirals. J Clin Oncol 2003;21:2876-82.

15. Spina M, Balzarotti M, Uziel L, et al. Modulated chemotherapy according to modified comprehensive geriatric assessment in 100 consecutive elderly patients with diffuse large B-cell lymphoma. Oncologist 2012;17:838-46.

16. Sganga F, Landi F, Ruggiero C, et al. Polypharmacy and health outcomes among older adults discharged from hospital: results from the CRIME study. Geriatr Gerontol Int 2015;15:141-6.

17. Berretta $M$, Tirelli $U$. The economic face to pharmacogenomic marker in cancer therapy. WCRJ 2014;1:e354. 